Document downloaded from:

http://hdl.handle.net/10251/43473

This paper must be cited as:

Nasir, S.; Ramirez Hoyos, P.; Ali, M.; Ahmed, I.; Fruk, L.; Mafé, S.; Ensinger, W. (2013). Nernst-Planck model of photo-triggered, pH-tunable ionic transport through nanopores functionalized with "caged" lysine chains. Journal of Chemical Physics. 138(3):034709-1. 034709-11. doi:10.1063/1.4775811.

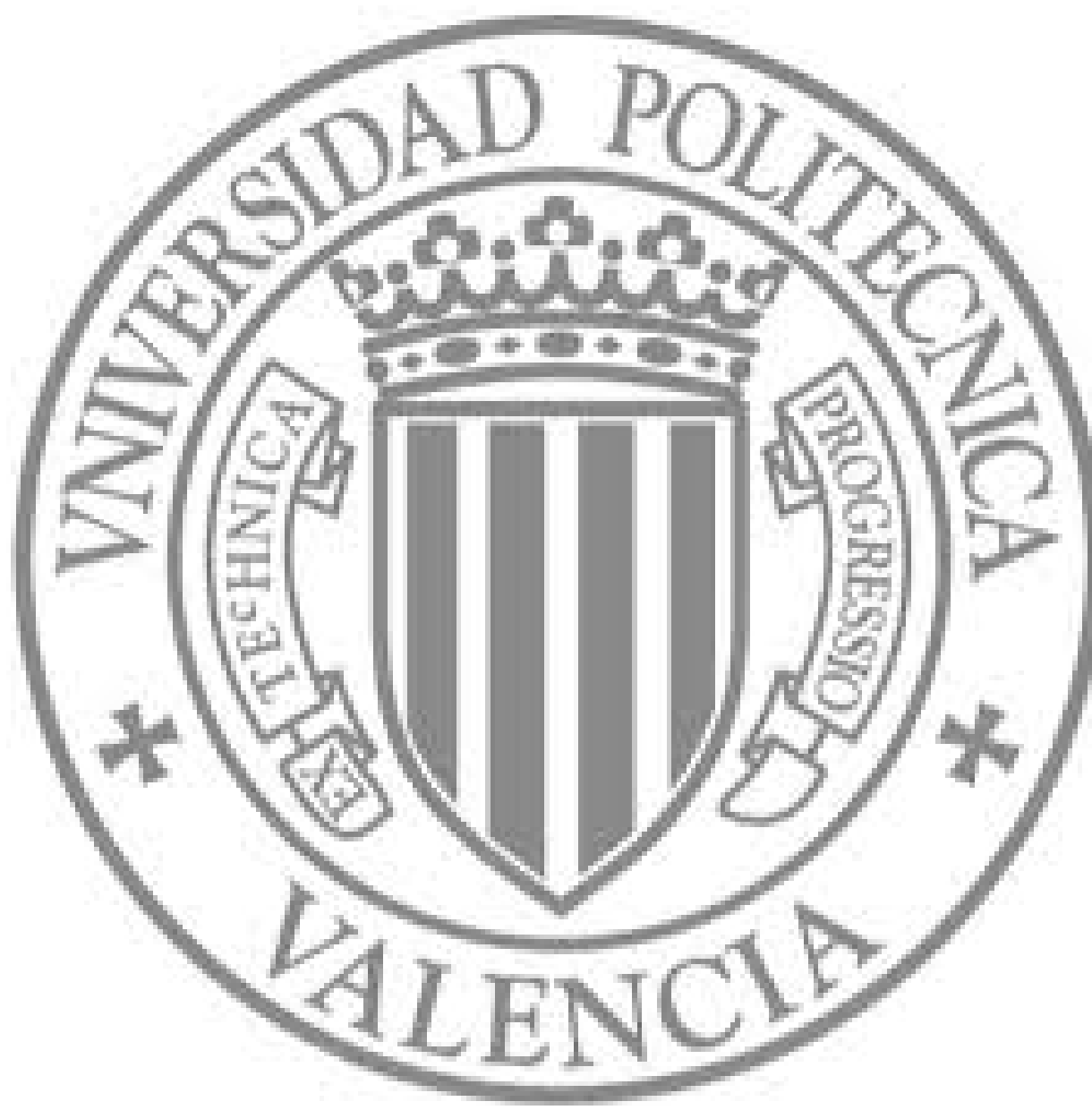

The final publication is available at

http://dx.doi.org/10.1063/1.4775811

Copyright American Institute of Physics (AIP) 


\title{
Nernst-Planck model of Photo-triggered, $p H$-Tunable Ionic Transport through Nanopores Functionalized with “Caged” Lysine Chains
}

\author{
Saima Nasir, ${ }^{1,2}$ Patricio Ramirez, ${ }^{3}$ Mubarak Ali, ${ }^{1,2, a)}$ Ishtiaq Ahmed, ${ }^{4}$ Ljiljana Fruk, \\ Salvador Mafe, ${ }^{5}$ and Wolfgang Ensinger ${ }^{1,2}$ \\ ${ }^{I}$ Department of Material- and Geo-Sciences, Materials Analysis, Technische Universität
} Darmstadt, D-64287 Darmstadt, Germany

${ }^{2}$ Materials Research Department, GSI Helmholtzzentrum für Schwerionenforschung, Planckstrasse 1, D-64291, Darmstadt, Germany

${ }^{3}$ Dept. de Física Aplicada. Univ. Politècnica de València. Camino de Vera s/n, E-46022 Valencia, Spain.

${ }^{4}$ Karlsruher Institute of Technology, DFG-Center for Functional Nanostructures Wolfgang-Gaede-Strasse 1, D-76131 Karlsruhe, Germany

${ }^{5}$ Dept. de Física de la Terra i Termodinàmica, Universitat de València, E-46100 Burjassot, Spain

${ }^{\text {a) Author to whom correspondence should be addressed. Electronic mail: m.ali@gsi.de }}$

\begin{abstract}
We describe the fabrication of asymmetric nanopores sensitive to ultraviolet (UV) light and give a detailed account of the divalent ionic transport through these pores using a theoretical model based on the Nernst-Planck equations. The pore surface is decorated with lysine chains having $\mathrm{pH}$-sensitive (amine and carboxylic acid) moieties that are caged with photo-labile 4,5-dimethoxy-2-nitrobenzyl (NVOC) groups. The uncharged hydrophobic NVOC groups are removed using UV irradiation, leading to the generation of hydrophilic "uncaged" amphoteric groups on the pore surface. We demonstrate experimentally that polymer membranes containing single pore and arrays of asymmetric nanopores can be employed for the $p H$-controlled transport of ionic and molecular analytes. Comparison between theory and experiment allows for understanding the individual properties of the
\end{abstract}


phototriggered nanopores and provides also useful clues for the design and fabrication of multipore membranes to be used in practical applications.

\section{INTRODUCTION}

Nanoscale pores have been studied extensively in the last decade due to the new basic phenomena involved and the potential applications in medicine, ${ }^{1-3}$ nanofluidics, ${ }^{4-7}$ membrane science $^{8,9}$ and biotechnology. ${ }^{10,11}$ Polymer samples containing single asymmetric nanopores and multipore arrays obtained by track-etching ${ }^{12}$ are of particular interest because they mimic some of the transport properties of biological ion channels. ${ }^{13-19}$ Recent advances concerning the fabrication processes and the tailoring of the surface properties have permitted to control the pore geometry ${ }^{20-23}$ and the response to external stimuli such as voltage, ${ }^{24,25}$ temperature, ${ }^{26,27} \mathrm{pH}^{28-30}$ or the presence of a given analyte in the pore solution. ${ }^{31-34}$ These features have allowed the fabrication of nanofluidic devices ${ }^{35-38}$ with potential applications in sensing, ${ }^{34,39,40}$ energy harvesting ${ }^{41,42}$ and information processing. ${ }^{07,42-44}$

Recently, synthetic nanopores sensitive to ultraviolet (UV) light have been reported. ${ }^{45,46}$ The pores use photo-labile protecting groups (PPGs) or photosensitive active groups, initially in hydrophobic state, that switch to a hydrophilic form after UV light irradiation. UV light constitutes a facile way to change the pore functionalities externally, without causing further damage to the active groups attached to the pore wall.

In this paper we describe a technique to obtain asymmetric nanopores sensitive to UV light. To achieve this goal, the "caged" lysine amino acid is chemically synthesized with an unprotected amine group at the terminus of alkyl chain. Then the pore surface and inner pore walls are functionalized with monolayers of amino acid "caged" lysine chains through carbodiimide coupling chemistry. The immobilized lysine chains contain photolysable 4,5dimethoxy-2-nitrobenzyl (NVOC) moieties attached to the $-\mathrm{NH}_{2}$ and $-\mathrm{COOH}$ groups at $\alpha-$ 
carbon of lysine. Upon UV treatment, the uncharged hydrophobic aromatic chromophore NVOC moieties are removed and hydrophilic "uncaged" amphoteric groups sensitive to environmental $\mathrm{pH}$ are exposed on the pore surface. The UV treated asymmetric nanopore displays a broad range of $p H$-controlled rectifying properties. Firstly, we demonstrate experimentally that polymer samples containing single and multiple UV light-operated pores can be employed for the $p H$-controlled transport of divalent anionic and cationic analytes across the membrane. Secondly, we present a detailed account of the experimental findings using a theoretical model based on the Nernst-Planck (NP) equations. The systematic comparison of the model predictions with the experimental data allows for the estimation of the pore characteristics and the diffusion coefficients of the divalent analytes. The approach provides an understanding of the properties of a single pore that should be useful in the design of multipore membranes for sensing, controlled release, and information processing.

\section{EXPERIMENTAL}

\section{Photosensitive “caged” amino acid lysine}

Single asymmetric nanopores (Fig. 1a) as well as multipore membranes containing arrays of pores were fabricated from heavy ion tracked polyethylene terephthalate (PET) membranes of thickness $12 \mu \mathrm{m}$ by well-established track-etching techniques. ${ }^{12}$ During the chemical etching process, the carboxylic acid groups generated on the pore surface act as sites for the covalent attachment of functional molecules containing primary amine in their backbone.

The photosensitive "caged" lysine amino acid with a free amine group at $\varepsilon$-position was synthesized following the chemical reactions shown in the scheme of Fig. 1b. The compound 3 was obtained in high yield by the esterification reaction between Fmoc-Lys(Boc)-OH (1)

and 6-nitroveratryl alcohol (2) using dicyclohexyl carbodiimide (DCC) and 4-(dimethyl 
amino)-pyridine (DMAP) in dichloromethane at room temperature. The deprotection of Fmoc-group in 3 yields compound 4 (Fig. 1b), which was used without purification in the next reaction step. The compound 6 was synthesized by the direct coupling reaction between deprotected amine of compound 4 with reactive 4,5-dimethoxy-2-nirobenzyl chloroformate 5 (NVOC-Cl). Finally, the deprotection of Boc-group in 6 afforded the desired lysine (7).

The "caged" lysine chains (7) of Fig. 1b were immobilized on the pore surface and inner walls through carbodiimide coupling chemistry. To this end, the track-etched membranes were treated with an activation solution containing $N$-(3-dimethylaminopropyl- $N^{\prime}-$ ethylcarbodiimide (EDC) and pentafluorophenol (PFP) to convert the native $-\mathrm{COOH}$ groups into amine-reactive ester molecules. Subsequently, the activated PFP-ester molecules were covalently coupled with the terminal $\varepsilon$-amine group of photosensitive lysine molecules.

\section{Single asymmetric nanopore}

The success of the pore surface modification reaction was confirmed by measuring the current-voltage $(I-V)$ characteristics of a single nanopore prior to and after the immobilization of "caged" lysine chains (Fig. 2). For the case of as-prepared nanopores, native ionized carboxylate $\left(-\mathrm{COO}^{-}\right)$groups impart negative charge to the pore walls under neutral and basic $p H$ conditions. Therefore, the asymmetric single pore shows rectification characteristics that depend on the surface charge of the pore walls, which can be tuned by the $p H$ value of the external solutions. By changing the solution $p H$ from basic to acidic values, a significant decrease in positive currents (from the pore tip to the pore basis) is observed, suggesting a decrease in the surface charge density due to the protonation of - $\mathrm{COOH}$ groups (Fig. 2a).

After functionalization, the hydrophobic and uncharged photolabile NVOC groups in the immobilized lysine chains drastically reduce the ionic current and rectification, as it is 
shown in the $I-V$ curve of Fig. 2b. This experimental fact confirms the successful anchoring of "caged" lysine chains onto the surface and walls of the pore.

Upon UV light irradiation of the pore, the NVOC groups are detached from the immobilized lysine chains and the amphoteric groups are exposed on the pore surface. This switches the chemical characteristics of the pore from hydrophobic to hydrophilic, which in turn changes the ion transport behavior (Fig. 2c) from non-selective ("off" state) to permselective ("on" state).

Due to the amphoteric nature of the resulting end groups, the $p H$ of the surrounding solutions dictates the pore net charge and permselectivity. The $I-V$ curves of Fig. 2c confirm the successful uncaging of the $-\mathrm{NH}_{2}$ and $-\mathrm{COOH}$ groups of lysine chains immobilized on the pore surface. At $p H=9.5$, both groups are deprotonated and the net charge on the pore surface is negative due to the presence of ionized $-\mathrm{COO}^{-}$groups. The pore is cation-selective and rectifies the ionic current flowing across the membrane. On the contrary, at acidic conditions $(p H=3.0)$ both groups are protonated and the $-\mathrm{NH}_{3}{ }^{+}$groups impart positive charge to the pore. Due to the switching of the pore net charge from negative to positive, the permselectivity and rectification behavior is also reversed (the pore is now anion-selective). Finally, at the intermediate $p H=5.0$, the pore behaves like an ohmic resistor because the net pore charge is zero due to the ionization of the $-\mathrm{COO}^{-}$and $-\mathrm{NH}_{3}{ }^{+}$groups (Fig. 2c). It is interesting to note that this behavior is present to some degree in the case of the $I-V$ curves corresponding to the pore with "caged" lysine chains (Fig. 2b). This fact reveals the presence of a small amount of residual "uncaged" amphoteric groups in the pore surface before UV irradiation. 


\section{Multipore membranes}

We have also investigated the UV-light-induced transport of divalent analytes across the nanoporous membrane containing $5 \times 10^{7}$ pores $\mathrm{cm}^{-2}$ functionalized with "caged" lysine chains (Fig. 3), prior to and after UV treatment. The membrane sample separated the feed compartment filled with an aqueous solution of either cationic or anionic analyte and the permeate compartment contains a buffer solution only. The results obtained are presented in Figs. 4 (methylviologen ion, $\mathrm{MV}^{2+}$ ) and 5 (1,5-naphthalene disulfonate ion, $\mathrm{NDS}^{2-}$ ). The flux of analyte through the membrane sample (Figs. $4 \mathrm{~d}$ and $5 \mathrm{~d}$ ) was obtained by monitoring the time-dependent concentration of analyte in the permeate chamber (Figs. 4a, 4b, 5a and 5b) from UV absorbance measurements with a UV/Vis spectrophotometer (Figs. 4c and 5c). The experiments clearly suggest that the transport of analyte is governed by the interaction of the permeate ions with the groups fixed on the pore surface.

Before UV irradiation (Fig. 3a, left), the pore is in hydrophobic ("off") state and the divalent ions in the external solutions can hardly enter and pass through the nanopores. The resulting analyte fluxes are therefore relatively small at all $p H$ values (Figs. $4 \mathrm{~d}$ and $5 \mathrm{~d}$ ). After UV irradiation (Fig. 3a, right) the pore switches to a hydrophilic ("on") state and the analyte fluxes depend markedly on the environmental $p H$ (Fig. 3b). Ionic species with the same sign as that of the pore charge are prevented from entering the membrane while species with opposite charge to that of the pore permeate through the pore. Finally, in the case of neutral pores both positive and negative species are almost equally transported across the pores, and the membrane is not-selective. Note that the pore with "caged" lysine chains (Figs. 4a and 5a) shows qualitatively similar $p H$-controlled transport properties as the pore with "uncaged" lysine groups (Figs. $4 b$ and $5 b$ ), which suggests again that some residual "uncaged" amphoteric groups are present in the pore surface before UV irradiation. 


\section{THEORY}

We consider the scheme of Fig. 6 where a polymeric film of thickness $d$ containing a single asymmetric nanopore separates two electrolyte solutions. The pore radius $a(x)$ has symmetry of rotation around the axis $x$ and can be described by the equation ${ }^{47,48}$

$$
a(x)=\frac{a_{R}-a_{L} \exp \left[-(d / h)^{n}\right]-\left(a_{R}-a_{L}\right) \exp \left[-(x / d)^{n}(d / h)^{n}\right]}{1-\exp \left[-(d / h)^{n}\right]} \quad(n>0),
$$

where $a_{L}$ and $a_{R}$ are, respectively, the radii at the left (tip) and right (base) pore openings. Typical nanopores obtained using the track-etching procedure show pore openings ranging from a few or tens of nanometers (pore tip) to a few hundreds of nanometers (pore base), while the pore length $d$ is of the order of $10 \mu \mathrm{m} .{ }^{12}$ Therefore, the pores can be assumed to be long and narrow, $d>>a(x)$. The radius profiles generated by Eq. (1) are in agreement with the typical tip shapes obtained by using recent improvements of the track-etching procedure. $^{21,2248-51}$ The pore shape can be controlled by changing the values of the geometrical parameters $n$ and $d / h$ in Eq. (1). ${ }^{47}$ For instance, $n=1$ and $d / h>0$ give profiles showing concave, bullet-like pore tips (the limit $d / h \rightarrow 0$ corresponds to the case of a conical pore). Using $d / h \rightarrow 0$ and $n>1$ lead to pores with convex, trumpet-like profiles. Finally, the values $d / h>0$ and $n>1$ produce profiles showing pore tips and lumens of variable length. ${ }^{47}$

In Fig. 6, $p H_{j}$ refers to the $p H$ value of solution $j(j=L$ for the left and $j=R$ for the right external solutions). In the following calculations, we assume $p H_{L}=p H_{R} \equiv p H$. Also, $c_{i, j}$ is the concentration of ion $i$ in the bulk of solution $j$ and $\phi_{j}$ denotes the dimensionless electric potential (normalized to $R T / F$, where $R, T$ anf $F$ have their usual meaning ${ }^{47}$ ) in the bulk of the solution $j$, respectively. Finally, $c_{i}$ and $\phi$ refer to the local concentration of species $i$ and dimensionless electric potential, respectively. The potential drops $\Delta \phi_{L}=\phi(0)-\phi_{L}$ and $\Delta \phi_{R}=$ 
$\phi_{R}-\phi(d)$, are the Donnan potential differences through the left and right interfaces, respectively. Finally, $\sigma$ is the surface charge density, assumed to be constant and related to the volume concentration of fixed charges $X_{F}$ as

$$
X_{F}=\frac{2 \sigma}{a F} \text {. }
$$

The external solutions are considered to be ideal and perfectly stirred (the effect of the diffusion boundary layers is ignored because the resistance to flow is due to the single nanopore) and the system is isothermal and at steady state. Finally, convective flows are ignored (note that the experiments of Figs. 4 and 5 are conducted under zero electric current).

We assume that the ionic transport through the pore can be described by the NernstPlanck equations

$$
\vec{J}_{i}=-D_{i}\left(\vec{\nabla} c_{i}+z_{i} c_{i} \vec{\nabla} \phi\right)
$$

the continuity equation at steady state

$$
\nabla \cdot \vec{J}_{i}=0
$$

and the Poisson equation

$$
\nabla^{2} \phi=-\frac{F^{2}}{\varepsilon R T}\left(\sum_{i} z_{i} c_{i}+X_{F}\right) .
$$

In Eqs. (3) - (5), $D_{i}, z_{i}$ and $\vec{J}_{i}$ are the diffusion coefficient, the charge number and the ionic flux density of ion $i$, respectively, and $\varepsilon$ is the electric permittivity of the aqueous solution (approximately equal to that of water).

As stated above, the pore is long and narrow, and the ionic fluxes can be assumed to have only axial component 
$\vec{J}_{i} \approx J_{i} \hat{u}_{x}$.

According with this approximation,

$j_{i} \approx-\pi a^{2} D_{i}\left(\frac{d c_{i}}{d x}+z_{i} c_{i} \frac{d \phi}{d x}\right)$,

where

$j_{i}=\pi a^{2} J_{i}$

is the total flux of ion $i$ through an arbitrary section of the pore (Eq. (1)). Equation (4) is then approximated as

$$
\frac{d j}{d x}=0
$$

Finally, the local electroneutrality condition

$\sum_{i} z_{i} c_{i}+X_{F}=0$

can be used instead of the Poisson equation in the case of long pores. Indeed, for onedimensional problems where averaging to the longitudinal channel axis is assumed, Eq. (10) can replace the Poisson equation when the axial pore length is much greater than the Debye length, ${ }^{52-54}$ which is the region where significant deviations from electroneutrality occur. The Debye length is typically $3-30 \mathrm{~nm}$ thick for most aqueous electrolyte solutions. Our pore is $12000 \mathrm{~nm}$ thick while the pore tip radii are in the $5 \mathrm{~nm}$ range. Therefore, Eq. (10) constitutes a useful approximation to the complex transport phenomena studied here.

The values of the electric potential and the ionic concentrations at the pore limits $x=0$ and $x=L$ are unknown and must be calculated in terms of those in the external solutions. Again, the influence of the access resistance can be neglected, because the pore is long and 
narrow, and we can assume the Donnan equilibria ${ }^{52,53,55}$ at the interfaces at $x=0$ and $x=d$ (Fig. 6):

$$
\begin{aligned}
& c_{i}(0)=c_{L} \exp \left(-z_{i} \Delta \phi_{L}\right), \\
& c_{i}(d)=c_{R} \exp \left(z_{i} \Delta \phi_{R}\right) .
\end{aligned}
$$

Combining Eqs. (10) and (11) with the electroneutrality condition in the bulk of the external solutions

$$
\sum_{i} z_{i} c_{i, j}=0, j=L, R
$$

allows for the calculation of the concentrations of all mobile ionic species at the pore limits, $c_{i}(0)$ and $c_{i}(d)$, and the interfacial Donnan potential differences (Fig. 6) at any value $p H_{j}$ Finally, Eqs. (7)-(10), together with the boundary conditions given in Eqs. (11)-(12), can be integrated using iterative schemes to obtain the concentrations and electric potential profiles, as well as the total fluxes, for any applied voltage $V \equiv R T\left(\phi_{L}-\phi_{R}\right) / F$. From the ionic fluxes, the total electric current passing through the pore is

$$
I=\sum_{i} z_{i} F j_{i}
$$

Note that Fig. 6 shows the sign criteria used for $V$ and $I$. Mathematically, the solution to the above transport equations could be obtained as an inverse problem where the structural characteristics of the nanopore should be determined from measurements of its function. ${ }^{56,57}$ However, we will use simple, approximate models that address the phenomenological basis of the observed phenomena.

\section{A single pore with $\mathrm{COOH}$ groups}

In the case of the as-prepared single nanopore with native - $\mathrm{COOH}$ groups, we have considered the following equilibrium between the neutral and ionized carboxylate groups 
$\mathrm{COOH} \stackrel{K_{C}}{\rightleftarrows} \mathrm{COO}^{-}+\mathrm{H}^{+}$,

The local fixed charge concentration in Eq. (2) can be written as

$X_{F}=-X_{C}^{-}=-\frac{X_{C}^{T}}{1+c_{H^{+}} / K_{C}}$,

where

$K_{C}=10^{-p K_{C}}=\frac{X_{C^{-} \mathrm{H}^{+}}^{-}}{X_{C}^{0}}$,

is the equilibrium constant of Eq. (14), $X_{C}^{-}$and $X_{C}^{0}$ are the volume concentration of carboxylic groups in ionized and neutral forms, respectively, and

$X_{C}^{T}=X_{C}^{-}+X_{C}^{0}$

is the total volume concentration of carboxylic groups.

\section{A single pore with amphoteric groups}

For a pore containing amphoteric groups, we have considered the equilibria

$$
\begin{aligned}
& \mathrm{NH}_{3}^{+}-\mathrm{A}-\mathrm{COOH} \stackrel{K_{A, 1}}{\rightleftarrows} \mathrm{NH}_{3}^{+}-\mathrm{A}-\mathrm{COO}^{-}+\mathrm{H}^{+}, \\
& \mathrm{NH}_{3}^{+}-\mathrm{A}-\mathrm{COO}^{-}+\stackrel{K_{A, 2}}{\rightleftarrows} \mathrm{NH}_{2}-\mathrm{A}-\mathrm{COO}^{-}+\mathrm{H}^{+},
\end{aligned}
$$

and the local volume fixed charge concentration of Eq. (2) is now

$$
X_{F}=X_{A}^{+}-X_{A}^{-}=\frac{X_{A}^{T}\left[c_{H^{+}}^{2} /\left(K_{A, 1} K_{A, 2}\right)-1\right]}{1+c_{H^{+}} / K_{A, 2}+c_{H^{+}}^{2} /\left(K_{A, 1} K_{A, 2}\right)},
$$

where 


$$
\begin{gathered}
K_{A, 1}=10^{-p K_{A, 1}}=\frac{X_{A}^{ \pm} c_{H^{+}}}{X_{A}^{+}}, \\
K_{A, 2}=10^{-p K_{A, 2}}=\frac{X_{A}^{-}{ }_{\mathrm{H}^{+}}}{X_{A}^{ \pm}},
\end{gathered}
$$

are the equilibrium constants in Eqs. (18) and (19), $X_{A}^{ \pm}, X_{A}^{-}$and $X_{A}^{+}$are the volume concentration of amphoteric groups in zwitterionic, negatively charged, and positively charged forms, respectively, and $X_{A}^{T}=X_{A}^{-}+X_{A}^{+}+X_{A}^{ \pm}$is the total volume concentration of amphoteric groups in the pore. For the sake of simplicity, we have assumed that the surface charge concentration in the pore is constant and equal to that calculated for the pore tip.

\section{Estimation of the pore parameters}

The pore parameters involved in the transport process were estimated according to the following protocol. The radius of the pore base was measured directly using AFM techniques in polymer samples with a high number of pores etched simultaneously with the sample containing the single nanopore. In our case, we obtained $a_{R} \approx 300 \mathrm{~nm}$. Because small variations in $a_{R}$ lead to negligible changes in the calculated $I-V$ curves, we employed this value in all calculations. We used also the free solution diffusion coefficients $D_{K^{+}}=1.9510^{-5}$ $\mathrm{cm}^{2} / \mathrm{s}, D_{\mathrm{Cl}^{-}}=2.0310^{-5} \mathrm{~cm}^{2} / \mathrm{s}, D_{H^{+}}=910^{-5} \mathrm{~cm}^{2} / \mathrm{s}$ and $D_{O H^{-}}=4.510^{-5} \mathrm{~cm}^{2} / \mathrm{s}^{58}$

When the pore is uncharged $\left(X_{F}=0\right.$ and $\sigma=0$ in the model), the $I-V$ curves depend only on the concentrations of mobile ions in the surrounding solutions and the geometrical characteristics of the pore. Under these conditions, the concentrations of mobile ions within the pore are constant,

$c_{i}=c_{i, L}=c_{i, R} \equiv c_{i, B}$, 
and integration of Eqs. (6)-(10) gives the linear $I-V$ curve

$I=\frac{\kappa}{g_{m}} V$

where

$\kappa=\frac{F^{2}}{R T} \sum_{i} z_{i}^{2} D_{i} c_{i, B}$

is the conductivity of the solution inside the pore and

$g_{m}=\int_{0}^{d} \frac{d x}{\pi a^{2}}$

is a geometrical factor depending exclusively on the pore shape (see Eq. (1) for $a(x)$ ). In the case of a conical pore, this factor gives

$g_{m}=\frac{d}{\pi a_{L} a_{R}}$

Note that for $a_{L}=a_{R}$, Eq. (27) gives the well-known result corresponding to the cylindrical pore (Fig. 1). For geometries other than cylindrical (or conical), Eq. (26) must be solved numerically taken into account the radius profile of Eq. (1).

In the experiments of Fig. 2, we obtained quasi-linear $I-V$ curves for the case of the pores containing "uncaged" and "caged" lysine groups when these chains are mostly in zwitterionic form (Figs. $2 \mathrm{~b}$ and $2 \mathrm{c}$ at $p H=5.0$ ). A linear fitting using the least square method provides the slopes (pore conductances) of the $I-V$ curves. Because the concentrations of all ionic species can be calculated from the electrolyte concentration and the $\mathrm{pH}$ of the external solutions, the only free parameter in the slope of the straight line is the geometrical factor $g_{m}$. The results obtained for the experimental data in Figs. $2 \mathrm{~b}$ and $2 \mathrm{c}$ are summarized in Table I. Once the value of $g_{m}$ has been determined, the radius of the pore tip can be calculated from 
Eq. (26) assuming a particular geometry in Eq. (1). Table I shows the values of $a_{L}$ obtained using this procedure. We have considered the cases of a perfectly conical pore and an asymmetric pore showing a convex profile that deviates slightly from the conical geometry $(d / h \rightarrow 0$ and $n=1.5$ in Eq. (1); see Fig. 6).

We conclude that the assumption of conical geometry gives unrealistically low values of $a_{L}$ for pores fabricated using the track-etching method..$^{33,48,59-61}$ If we assume convex pores, the values of $a_{L}$ in Table I are now within the range of those estimated in previous studies. ${ }^{33,48,59-61}$ Otherwise stated, we will assume thus that the pore tip is convex and deviates slightly from the conical geometry. As expected, the effective pore radii obtained for the pores with "caged" lysine chains are significantly lower than those corresponding to the "uncaged" chains (Table I), in agreement with the bulky terminal ends and its hydrophobic character (Fig. 2a).

We consider now the case of charged pores. The results obtained for the as-prepared pore with carboxylate groups (Fig. 2a) show non-linear curves at the $p H$ values measured and thus the above procedure cannot be applied directly. Since the value obtained for $a_{L}$ in the case of the pores with "uncaged" lysine reveals a relatively wide tip opening, we can assume that the pore tip radius of the as-prepared nanopore is the same than that calculated from Fig. $2 \mathrm{c}, a_{L}=5.1 \mathrm{~nm}$. With this assumption, the only free parameters in the NP model are the surface charge density $\sigma$ and the $p K_{a}$ value of the carboxylate groups, $p K_{C}$. The best fit for the as-prepared pore (Fig. 7a) gave a surface charge density $\sigma=-0.3 e \mathrm{~nm}^{-2}$ at $p H=9.5$, where $e$ is the elementary charge, and $p K_{C}=4.5$. Because at $p H=9.5$ all the carboxylate groups are in charged form, it can be concluded that the surface density of groups attached to the pore walls is approximately $n_{C}=0.3$ groups $/ \mathrm{nm}^{2}$. The values of $n_{C}$ and $p K_{C}$ are in agreement with those found in previous studies for the case of pores functionalized with carboxylate groups. ${ }^{33,61}$ 
It must also be mentioned that similar calculations assuming a perfect conical geometry instead of a convex tip geometry lead to unrealistically low values of the surface charge density $\left(\sigma=-0.07 e \mathrm{~nm}^{-2}\right.$ at $p H=9.5$ in the case of the experiments of Fig. 2a). Also, the rectification ratios (defined as the absolute value of the ratio between the electric current in the "on" and "off" states at a given voltage) calculated assuming a perfect conical geometry are much higher than those obtained using the experimental data of Fig. 2a. These two facts give further support to our assumption that the pore with a convex tip deviates slightly from the conical geometry.

We consider now the experiments with the pore functionalized with "uncaged" lysine chains (Fig. 2c). Because the axial pore profile and the values of the pore radii at the tip and base have already been determined, the unknown parameters in the NP model are the surface density $n_{A}$ and the two $p K_{a}$ values of the amphoteric lysine groups, $p K_{A, 1}$ and $p K_{A, 2}$. Figure 7c shows the theoretical results obtained for this pore using $n_{A}=0.3$ groups $/ \mathrm{nm}^{2}, p K_{A, 1}=3$ and $p K_{A, 2}=9$. The value for $n_{A}$ assumes a $100 \%$ substitution of the original carboxylate groups by the amphoteric groups. The values for $p K_{A, 1}$ and $p K_{A, 2}$ provide the best fit to the experimental data of Fig. 2c and are close to the $p K_{a}$ values of lysine in free solution $(2.18$ and 8.95$){ }^{62}$ which gives support to the pore characterization procedure followed here.

Finally, Fig. 7b shows the results provided by the NP model for the pore functionalized with "caged" lysine. Using the $p K_{A, 1}$ and $p K_{A, 2}$ values calculated above, the best fit to experimental data was obtained assuming $n_{A}=0.12$ groups $/ \mathrm{nm}^{2}$. This value suggests that approximately one third of the supposedly "caged" lysine groups were actually in ionized form, which explains the residual $p H$-controlled transport shown in Figs. $2 \mathrm{~b}$ and $7 \mathrm{~b}$. 


\section{Permeation of $\mathrm{MV}^{2+}$ and $\mathrm{NDS}^{2-}$}

The ionic analyte molecules used in the transport measurements through the multipore membranes of Figs. 4 and 5 were methylviologen dichloride $\left(\mathrm{MVCl}_{2}\right)$ and 1,5-naphthalene disulfonate di-sodium salt ( $\left.\mathrm{Na}_{2} \mathrm{NDS}\right)$. The ionic species involved in the permeation process were $\mathrm{MV}^{2+}$ and $\mathrm{Cl}^{-}$in the case of the methylviologen dichloride and $\mathrm{NDS}^{2-}$ and $\mathrm{Na}^{+}$for the 1,5-naphthalene disulfonate di-sodium salt. For the sake of simplicity, we have neglected the influence of the ionic species present in the buffer solutions used to control the external $p H$. Also, we restrict our calculations to the case of the membrane in the "on" state (after UV irradiation) when all the lysine groups are "uncaged".

In the case of $\mathrm{MVCl}_{2}$ we consider again the case $X_{F}=0(p H=5.0)$. When the membrane is uncharged, integration of the Nernst-Planck equations gives the total flux

$j_{\mathrm{MV}^{2+}} n_{p} A_{\text {sample }}=\frac{3 c_{\mathrm{MV}^{2+}}(0)}{g_{m}\left(1 / D_{\mathrm{MV}^{2+}}+2 / D_{\mathrm{Cl}^{-}}\right)} n_{p} A_{\text {sample }}$,

where $n_{p}$ is the number of pores per unit of area and $A_{\text {sample }}$ is the membrane area used in the experiments. Equation (28) is obtained with the condition of zero electric current through the membrane

$2 j_{\mathrm{MV}^{2+}}-j_{\mathrm{Cl}^{-}}=0$.

Because the multipore membrane sample was etched at the same time as the single nanopore sample, we can assume that the geometrical factor $g_{m}$ is the same for the two samples. With this assumption, the only free parameter in Eq. (28) is the diffusion coefficient of the analyte. Using the experimental data of Fig. 4 for $p H=5.0$, we obtain $D_{\mathrm{MV}^{2+}}=5.110^{-7} \mathrm{~cm}^{2} / \mathrm{s}$. Similar calculations for the $\mathrm{NDS}^{2-}$ experimental data of Fig. $5 \mathrm{~d}$ at $p H=5$ give $D_{\mathrm{NDS}^{2-}}=2.510^{-7}$ 
$\mathrm{cm}^{2} / \mathrm{s}$. These values are in good agreement with those found in previous studies of $\mathrm{MVCl}_{2}$ and $\mathrm{Na}_{2}$ NDS diffusion through nanoporous membranes. ${ }^{45,63}$

In the case of acidic or basic $p H$, the membrane is charged and the Nernst-Planck equations must be solved using numerical procedures. In order to find an approximate analytical solution, we assume

$\pi a^{2} \frac{d \phi}{d x}=$ constant

Equation (30) is a generalization of the Goldman constant field assumption ${ }^{64}$ commonly used in the study of biological ion channels and synthetic membranes. ${ }^{65}$ With this assumption, the integration of the Nernst Planck equations yields

$j_{\mathrm{MV}^{2+}} n_{p} A_{\text {sample }}=\frac{2 \mathrm{MV}^{2+}{ }^{c} \mathrm{MV}^{2+}(0) u^{2} \log (1 / u)}{g_{m}\left(1-u^{2}\right)} n_{p} A_{\text {sample }}$,

where

$u=\frac{f_{c}+\sqrt{f_{c}^{2}+4 f_{c}}}{2}$

and

$f_{c}=\frac{D_{\mathrm{Cl}^{-}}}{4 D_{\mathrm{MV}^{2+}}} \frac{{ }_{\mathrm{Cl}^{-}}(0)}{{ }_{\mathrm{MV}^{2+}}(0)}$

Similar calculations allow the estimation of the total $\mathrm{NDS}^{2-}$ flux through the multipore membrane.

Because the concentration of the mobile species at the pore tip can be calculated from the external concentrations in the feeding compartment using Eqs. (10)-(12), the simplified model of Eqs. (30)-(33) contains no additional free parameters, and can then be used to check 
further the validity of the theoretical approach proposed here. The comparison between the model predictions and the experiments of Figs. 4 and 5 is given in terms of the ratio between the analyte fluxes in Table II. In spite of the rough approximations introduced, the agreement between theory and experiment is good for analytes with charge of the same sign as that of the pore surface charge. However, the theoretical model overestimates the fluxes if the analyte and surface charges have opposite signs. This result suggests that other effects in addition to those characteristic of the simple point ion models ${ }^{66}$ could be important for the relatively bulky divalent ions used in the permeation experiments.

\section{CONCLUSIONS}

Photochemical gating of nanoscale pores constitutes a subject of current interest because of the potential applications. ${ }^{45,46,67}$ We have described the transport properties of asymmetric nanopores functionalized with photosensitive amphoteric lysine groups. The experiments concern the $I-V$ curves of polymer samples containing the as-prepared single asymmetric nanopore with carboxylate groups acting as fixed charges, the $I-V$ curves of the single nanopore functionalized with "caged" and "uncaged" lysine groups, and the fluxes of divalent positive and negative analytes through multipore membranes. A detailed theoretical study based on the NP equations allows for obtaining the key parameters involved in the transport processes (pore tip shape and dimensions, surface charge concentrations, and $p K_{\mathrm{a}}$ values of the functional groups fixed on the pore walls). We have shown that a relatively simple continuum model involving a reduced number of basic concepts approximately describes the observed transport phenomena in terms of the electrostatic, $p H$-tunable interaction of the mobile ions with the pore surface charges. 


\section{ACKNOWLEDGEMENTS}

The authors would like to thank Miguel Ferrandez and Juan Pablo Arranz for assistance in the preparation of the artwork. P. R. and S. M. acknowledge financial support from the Ministry of Science and Innovation (projects Nos. MAT2009-07747 and MAT2012-32084) and the Generalitat Valenciana (project PROMETEO/GV/0069) and FEDER. S. N., M. A. and W. E. gratefully acknowledge financial support by the Beilstein-Institut, Frankfurt/Main, Germany, within the research collaboration NanoBiC, and L.F. and I.A. DFG-CFN Excellence Initiative Project A5.7. The authors thank Dr. Christina Trautmann from GSI (Materials research group) for support with the heavy ion irradiation experiments, and Dr. M.N. Tahir (Mainz University) for fruitful discussions and help in performing the UV light irradiation experiments. 


\section{REFERENCES}

${ }^{1}$ K. Healy, Nanomedicine 2, 459 (2007).

${ }^{2}$ J. Griffiths, Anal. Chem. 80, 23(2008).

${ }^{3}$ T. Jovanovic-Talisman, J. Tetenbaum-Novatt, A. S. McKenney, A. Zilman, R. Peters, M. P. Rout, and B. T. Chait, Nature 457, 1023 (2009).

${ }^{4}$ R. B. Schoch, J. Han, and P. Renaud, Rev. Mod. Phys. 80, 839 (2008).

${ }^{5}$ S.-W. Nam, M. J. Rooks, K.-B. Kim, and S. M. Rossnagel, Nano Lett. 9, 2044 (2009).

${ }^{6}$ J. M. Perry, K. Zhou, Z. D. Harms, and S. C. Jacobson, ACS Nano 4, 3897 (2010).

${ }^{7}$ W. Guan, R. Fan, and M. A. Reed., Nat. Commun. 2, 506 (2011).

${ }^{8}$ C. C. Striemer, T. R. Gaborski, J. L. McGrath, and P. M. Fauchet, Nature 445, 749 (2007).

${ }^{9}$ A. van den Berg, and M. Wessling, Nature 445, 726 (2007).

${ }^{10}$ C. Dekker, Nature Nanotechnology 2, 209 (2007).

${ }^{11}$ M. D. Mager, and N. A. Melosh, Adv. Mater. 20, 4423 (2008).

${ }^{12}$ P. Y. Apel, Y. E. Korchev, Z. Siwy, R. Spohr, and M. Yoshida, Nucl. Inst. Meth. B 184, 337 (2001).

${ }^{13}$ Z. Siwy, and A. Fuliński, Phys. Rev. Lett. 89, 198103 (2002).

${ }^{14}$ P. Ramírez, S. Mafé, V. M. Aguilella, and A. Alcaraz, Phys. Rev. E 68, 011910 (2003).

${ }^{15}$ Z. Siwy, and A. Fuliński, Am. J. Phys. 72, 567 (2004).

${ }^{16}$ Z. Siwy, I. D. Kosińska, A. Fuliński, and C. R. Martin, Phys. Rev. Lett., 94, 048102 (2005).

${ }^{17}$ M. R. Powell, M. Sullivan, I. Vlassiouk, D. Constantin, O. Sudre, C. C. Martens, R. S. Eisenberg, and Z. S. Siwy, Nat. Nanotechnol. 3, 51 (2008), 51.

${ }^{18}$ E. Garcia-Gimenez, A. Alcaraz, V. M. Aguilella, and P. Ramirez, J. Membrane Sci. 331, 137 (2009).

${ }^{19}$ X. Hou, H. Zhang, and L. Jiang, Angew. Chem. Int. Ed. 51, 5296 (2012).

${ }^{20}$ C. C. Harrell, Z. S. Siwy, and C. R. Martin, Small 2, 194 (2006). 
${ }^{21}$ P. Y. Apel, I. V. Blonskaya, S. N. Dmitriev, O. L. Orelovitch, A. Presz, and B. A. Sartowska, Nanotechnology 18, 305302 (2011).

${ }^{22}$ P. Y. Apel, I. V. Blonskaya, O. L. Orelovitch, P. Ramirez, and B. A. Sartowska, Nanotechnology 22, 175302 (2011).

${ }^{23}$ M. Ali, P. Ramirez, H. Q. Nguyen, S. Nasir, J. Cervera, S. Mafe, and W. Ensinger, ACS Nano 6, 3631 (2012).

${ }^{24}$ E. B. Kalman. O. Sudre, I. Vlassiouk, and Z. S. Siwy Anal. Bioanal. Chem. 394, 413 (2009).

${ }^{25}$ S. Mafe, J. A. Manzanares, and P. Ramirez, J. Phys. Chem. C 114, 21287 (2010)

${ }^{26}$ S. Nasir, M. Ali, and W. Ensinger, Nanotechnology 23, 225502 (2012).

${ }^{27}$ W. Guo , H. Xia , L. Cao, F. Xia, S. Wang, G. Zhang, Y. Song, Y. Wang, L. Jiang, and D. Zhu, Adv. Funct. Mater. 20, 3561 (2010).

${ }^{28}$ M.Ali, P. Ramirez, S. Mafe, R. Neumann, and W. Ensinger, ACS Nano 3, 603 (2009).

${ }^{29}$ M. Ali, S. Mafe, P. Ramirez, R. Neumann, and W. Ensinger, Langmuir 25, 11993 (2009).

${ }^{30}$ X. Hou, Y. Liu, H. Dong, F. Yang, L. Li, and L. Jiang, Adv. Mater. 22, 2440 (2010).

${ }^{31}$ X. Hou, W. Guo, F. Xia, F.-Q. Nie, H. Dong, Y. Tian, L. Wen, L. Wang, L. Cao, Y. Yang, J. Xue, Y. Song, Y. Wang, D. Liu, and L. Jiang, J. Am. Chem. Soc. 131, 7800 (2009).

${ }^{32}$ Y. He, D. Gillespie, D. Boda, I. Vlassiouk, R. S. Eisenberg, and Z. S. Siwy, J. Am. Chem. Soc. 131, 5194 (2009).

${ }^{33}$ M. Ali, R. Neumann, and W. Ensinger, ACS Nano 4, 7267 (2010).

${ }^{34}$ M. Ali, M. N. Tahir, Z. Siwy, R. Neumann, W. Tremel, and W. Ensinger, Anal. Chem. 83, 1673 (2011).

${ }^{35}$ I. Vlassiouk, and Z. Siwy, Nano Lett. 7, 552 (2007).

${ }^{36}$ E. B. Kalman, I. Vlassiouk, and Z. Siwy, Adv. Mater. 20, 293 (2008). 
${ }^{37}$ M. Ali, P. Ramirez, M. N. Tahir, S. Mafe, Z. Siwy, R. Neumann, W. Tremel, and W. Ensinger, Nanoscale 3, 1894 (2011).

${ }^{38}$ X. Hou, F. Yang, L. Li, Y. Song, L. Jiang, and D. Zhu, J. Am. Chem. Soc. 132, 11736 (2010).

${ }^{39}$ K. Healy, B. Schiedt, and A. P. Morrison, Nanomedicine 2, 875 (2007).

${ }^{40}$ C. R. Martin, and Z. S. Siwy, Science 317, 331 (2007).

${ }^{41}$ W. Guo, L. Cao, J. Xia, F.-Q. Nie, W. Ma, J. Xue, Y. Song, D. Zhu, Y. Wang, and L. Jiang, Adv. Funct. Mater. 20, 1339 (2010).

${ }^{42}$ J. Cervera, P. Ramirez, S. Mafe, and P. Stroeve, Electrochimica Acta 56, 4504 (2011).

${ }^{43}$ P. Ramirez, M. Ali, W. Ensinger, and S. Mafe, Appl. Phys. Lett. 101, 133108 (2012).

${ }^{44}$ Y. Jiang, N. Liu, W. Guo, F. Xia, and L. Jiang, J. Am. Chem. Soc. 134 , 15395. (2012)

${ }^{45}$ M. Ali, S. Nasir, P. Ramirez, I. Ahmed, Q. H. Nguyen, L. Fruk, S. Mafe, and W. Ensinger, Adv. Funct. Mater. 22, 390 (2012).

${ }^{46}$ M. Zhang, X. Hou, J. Wang, Y. Tian, X. Fan, J. Zhai, and L. Jiang, Adv. Mater. 24, 2424 (2012).

${ }^{47}$ P. Ramirez, P. Y. Apel, J. Cervera, and S. Mafe, Nanotechnology 19, 315707 (2008).

${ }^{48}$ M. Ali, B. Yameen, J. Cervera, P. Ramirez, R. Neumann, W. Ensinger, W. Knoll, and O. Azzaroni, J. Am. Chem. Soc. 132, 8338 (2010).

49 P.Y. Apel, I. V. Blonskaya, O. L. Orelovitch, B. A. Sartowska, and R. Spohr, Nanotechnology 23, 225503 (2012)

${ }^{50}$ N. Li, S. Yu, C. C. Harrell, and C. R. Martin, Anal. Chem. 76, 2025 (2004).

${ }^{51}$ C. C. Harrell, P. Kohli, Z. Siwy, and C. R. Martin, J. Am. Chem. Soc. 126, 15646 (2004).

52 J. A. Manzanares, S. Mafe, and J. Pellicer, J. Chem. Soc. Faraday Trans. 88, 2355 (1992).

${ }^{53}$ A. D. MacGillivray, J. Chem. Phys. 48, 2903 (1968). 
${ }^{54}$ I. Rubinstein, Electro-Diffusion of Ions. (SIAM Studies in Applied Mathematics, SIAM, Philadelphia, 1990).

${ }^{55}$ K. Kontturi, L. Murtomäki, and J. A. Manzanares, Ionic Transport Processes (Oxford University press, Oxford, 2008).

${ }^{56}$ M. Burger, Inverse Problems 2, 083001 (2011).

${ }^{57}$ M. Burger, R. S. Eisenberg, and H. Engl, SIAM J. Applied Math. 67, 960 (2007).

58 R. A. Robinson and R. H. Stokes, Electrolyte Solutions (Butterworth Scientific Publications, London, 1955).

${ }^{59}$ J. Cervera, B. Schiedt, and P. Ramírez, Europhys. Lett. 71, 35 (2005).

${ }^{60}$ J. Cervera, B. Schiedt, R. Neumann, S. Mafe, and P. Ramirez J. Chem. Phys. 124, 104706 (2006).

${ }^{61}$ J. Cervera, A. Alcaraz, B. Schiedt, R. Neumann, and P. Ramirez, J. Phys. Chem. C, 111, 12265 (2007).

${ }^{62}$ C. K. Mathews and K. E. Van Holde, Bioelectrochemistry (Benjamin/Cummings, Redwood City, CA, 1990).

${ }^{63}$ S. B. Lee, and C. R. Martin, Anal. Chem. 73, 768 (2001).

${ }^{64}$ J. Pellicer, S. Mafe, and V. M. Aguilella, Ber. Bunsenges. Phys. Chem. 90, 867 (1986).

${ }^{65}$ N. Lakshminarayanaiah, Equations of Membrane Biophysics (Academic Press, New York, 1984).

${ }^{66}$ J. Cervera, P. Ramirez, J. A. Manzanares, and S. Mafe, Microfluid. Nanofluid. 9, 41 (2010).

${ }^{67}$ G. L. Wang, A. K. Bohaty, I. Zharov, and H. S. White, J. Am. Chem. Soc. 128, 13553 (2006). 


\section{FIGURE CAPTIONS}

\section{Fig. 1:}

a) Scheme (not to scale) of the asymmetric nanopore (Fig. 1a). b) Reaction scheme for the synthesis of "caged" amino acid lysine (7) with caged amine and carboxylic acid groups attached to the $\alpha$-carbon (Fig. $1 b$ ).

\section{Fig. 2:}

Schematic pore and $p H$-dependent $I-V$ curves of a single asymmetric nanopore with carboxylate groups (Fig. 2a), "caged" (Fig. 2b), and "uncaged” amphoteric lysine chains (Fig. 2c). The $I-V$ curves are measured in a $0.1 \mathrm{M} \mathrm{KCl}$ solution prepared in a phosphate buffer at different $p H$ values.

\section{Fig. 3:}

Schematic cartoon describing the phototriggered permeation through the asymmetric nanopore arrays bearing "caged" lysine chains ("off" state) and "uncaged" lysine chains ("on" state) on the inner pore walls (Fig. 3a). The $p H$-tunable permselective transport of ionic analytes across the multipore membrane containing "uncaged" amphoteric lysine chains (Fig. $3 b)$.

\section{Fig. 4:}

$p H$-dependent permeation of $\mathrm{MV}^{2+}$ through the multipore membrane prior to (Fig. 4a) and after (Fig. 4b) UV irradiation. Absorption spectra recorded for $\mathrm{MV}^{2+}$ in the permeate solution obtained after 4 hours of analyte transport at $p H=9.5$ before and after UV irradiation of the membrane (Fig. 4c). $\mathrm{MV}^{2+}$ permeation rates through the multipore membrane before and after UV treatment, respectively (Fig. 4d). 


\section{Fig. 5:}

$p H$-dependent permeation of $\mathrm{NDS}^{2-}$ through the multipore membrane prior to (Fig. 5a) and after (Fig. 5b) UV irradiation. Absorption spectra recorded for $\mathrm{NDS}^{2-}$ in the permeate solution obtained after 4 hours of analyte transport at $p H=3.0$ before and after UV irradiation of the membrane (Fig. 5c). NDS $^{2-}$ permeation rates through the multipore membrane before and after UV treatment, respectively (Fig. 5d).

\section{Fig. 6:}

Sketch of the asymmetric nanopore (not to scale).

\section{Fig. 7:}

Calculated $I-V$ curves of a single asymmetric nanopore with carboxylate groups (Fig. 7a), "caged" lysine chains (Fig. 7b), and "uncaged" lysine chains (Fig. 7c), for the same experimental conditions as in Fig. 2. The nanopore parameters used in the calculations are shown in the insets.

\section{TABLE I:}

Estimation of the pore parameters from least-square fittings of the linear $I-V$ curves in Figs. $2 \mathrm{~b}$ and $2 \mathrm{c}$.

\section{TABLE II:}

The flux ratios at different $p H$ values obtained with the NP model and the experimental ratios for the permeation of $\mathrm{MV}^{2+}$ and $\mathrm{NDS}^{2-}$ through the multipore membrane. 